\title{
Relationship between red cell distribution width and mean platelet volume with new onset atrial fibrillation after off-pump coronary artery bypass grafting
}

\author{
Ozsin KK, Sanri US, Toktas F, Yavuz S \\ University of Health Sciences, Bursa Yuksek Ihtisas Training and Research Hospital, Yildirim, \\ Bursa, Turkey. kkozsin@gmail.com
}

\begin{abstract}
OBJECTIVE: The aim of this study was to evaluate the relationship between red blood cell distribution width (RDW) and mean platelet volume (MPV) with development of postoperative atrial fibrillation (PoAF) after offpump coronary artery bypass grafting (CABG).

BACKGROUND: The RDW and MPV have been associated with some cardiovascular disorders.

METHODS: A total of 93 patients who underwent off-pump CABG were included in this study. The patients were divided into two groups as developing and nondeveloping PoAF groups in the postoperative period. We measured whether RDW and MPV levels are a predictive value for development PoAF.

RESULTS: There were 24 patients with PoAF enrolled (mean age: $66 \pm 7.8$ years) and 69 patients without PoAF (mean age: $56.26 \pm 11.53$ years). The PoAF was significantly correlated with age $(p=0.004)$, hematocrit (p: 0.010$)$, RDW $(p=0.007)$ and creatinine $(p=0.006)$. Only advanced age $(p=0.012)$ was identified as an independent predictor of PoAF. For predicting PoAF, there was $79.2 \%$ sensitivity and $65.2 \%$ specificity for RDW $(p=0.001)$ and $62.5 \%$ sensitivity and $55.1 \%$ specificity for MPV $(p=0.062)$.

CONCLUSIONS: We found that RDW levels and MPV were not an independent predictor of the development of PoAF. However, elevated RDW levels and MPV may be one of the predictive values for PoAF development (Tab. 3, Fig. 2, Ref. 27). Text in PDF www.elis.sk.

KEY WORDS: red blood cell, mean platelet volume, atrial fibrillation, coronary artery bypass, off-pump.
\end{abstract}

\section{Introduction}

After coronary artery bypass grafting surgery $(\mathrm{CABG})$, atrial fibrillation (AF) may occur as a common complication (1). Postoperative atrial fibrillation (PoAF) has a high prevalence, affecting 20 to $45 \%$ of CABG (2). Atrial fibrillation frequently occurs in the first five days of the postoperative period and peaks between 24 and 72 hours. It becomes uncommon after the first week (2). PoAF has been associated with an increase in early and late mortality rates, hospital adverse events, particularly hemodynamic instability, thromboembolic events and heart failure progression.

Intraoperative factors can be consequent upon cardiac ischemia and inflammation due to the complexity of the surgical procedure. The CABG with cardiopulmonary bypass seems to have a higher incidence for PoAF as compared with off-pump CABG (2).

The red blood cell distribution width (RDW) is a test showing the change in size or volume of erythrocytes. It is expected that

University of Health Sciences, Bursa Yuksek Ihtisas Training and Research Hospital, Yildirim, Bursa, Turkey

Address for correspondence: K.K. Ozsin, MD, University of Health Sciences, Bursa Yuksek Ihtisas Training and Research Hospital, Prof. Tezok Street 152 Evler postal zip code: 16320, Yildirim, Bursa, Turkey.

Phone: +905326272833, Fax: +902242955497 the sizes of erythrocytes produced in the bone marrow are close to each other. It is not normal if there are differences between the sizes. Increases of RDW levels are correlated with inflammatory markers and are accepted as a sign of ineffective erythropoiesis which occurs in critically ill patients (3). In the last decade, studies have been carried out on prognostic marker on RDW in various cardiovascular disorders besides blood diseases. Many studies have shown that RDW is a strong predictor of adverse outcomes in patients with heart failure, stroke and myocardial infarction (4-6). In previous studies, in patients undergoing coronary angiography and $\mathrm{CABG}$ indicated a possible association between RDW and $\operatorname{AF}(7,8)$.

The mean platelet volume (MPV) is one of the most important biomarkers of platelet activity. Activated platelets have larger volumes and contain wide range of vasoactive substances and prothrombotic factors. Therefore, MPV may also be a response to inflammation and thrombosis (9). An increased MPV is associated with overall cerebrovascular and cardiovascular mortality rates (10).

The red blood cell distribution width and MPV have been associated with some cardiovascular disorders, but the relationship with $\mathrm{AF}$ is still uncertain. However, the relation between the RDW and MPV levels and PoAF has not been researched in patients undergoing off-pump coronary artery bypass grafting. We aimed to 
investigate the relation between the RDW and MPV levels with PoAF in these patients.

\section{Materials and methods}

\section{Patients}

This retrospective observational study included 114 consecutive patients who underwent off-pump CABG between 2014 and 2017 at the Department of Cardiovascular Surgery, Bursa Yuksek Ihtisas Training and Research Hospital, Bursa, Turkey. The study was approved by the local institutional Ethical Committee of University of Health Sciences.

All data to be analyzed retrospectively were retrieved from the hospital medical files. The exclusion criteria were preoperative AF or flutter, previous treatment with amiodarone, presence of valvular heart disease, chronic obstructive pulmonary disease (COPD), prolonged intensive care unit (ICU) stay, redo cardiac surgery, bleeding revision, chronic renal failure. Six patients with COPD, 2 patients with chronic renal failure, 7 patients with moderate mitral valve disease, 2 patients with preoperative AF, 2 patients with previous cardiac surgery history, 1 patient with postoperative bleeding and 1 patient with prolonged ICU stay were excluded. Thus, the remaining 93 patients with off-pump CABG were included in the study.

All data were recorded as age, gender, history of hypertension, diabetes mellitus, preoperative drug use (beta-blockers, statins, ACE or ARB inhibitors), ejection fraction, left atrial diameter, body mass index, body surface area, Euroscore, number of anastomoses, postoperative bleeding amount, duration of ICU stay, and discharge time from hospital.

\section{Laboratory measurements}

Fasting blood samples were drawn from an antecubital vein of each patient before operation. The tubes with EDTA were used for automatic blood count according to the protocol of our hospital. Complete blood cell count include parameters such as red blood cell count, hematocrit, hemoglobin, red cell distribution withd, mean corpuscular volume, total white blood cell count, neutrophil and lymphocyte counts, platelet count and mean platelet volume. These parametres were measured by using an automated hematological analyzer (Coulter LH 780 Analyzer, CA, USA). The complete blood cell parameters were measured through Coulter Erythrolyse II Reagent Kit (Beckman Coulter, Ireland). The reference range for RDW was $11.5-14.0 \%$, reference range for MPV was $6.96-10.13 \%$.

\section{Diagnosis of PoAF}

The patients were monitored in ICU with continous heart rhythm and invasive blood pressure monitoring. In addition, a 12-lead electrocardiographic recordings (ECG) were also obtained daily during in the ICU. Patients were monitored continuously by five-lead telemetry in the regular ward. When the patients complained of palpitation, dyspnea and angina, 12-lead ECG was taken. Postoperative AF was described as irregular, fast oscillations, or fibrillatory waves instead of regular P waves at ECG. An
AF episode lasting longer than 5 minutes was accepted as PoAF. Standard medical cardioversion treatment was conducted with amiodarone $(5 \mathrm{mg} / \mathrm{kg}$ ) for 30 minutes, followed by $900 \mathrm{mg} /$ day.

\section{Statistical analysis}

Statistical analysis data were analyzed with the Statistical Package for the Social Sciences (IBM SPSS Statistic Inc. version 21.0, Chicago, IL, USA).Continuous and ordinal variables were expressed as mean \pm standard deviation and nominal variables were expressed as frequency and percentage. Kolmogorov-Smirnov test and Shapiro-Wilk tests of normality were used to identify distribution of variables. Student's t test was used to compare two groups for continuous variables with normal distribution.Chi Square test was used to compare two groups for nominal variables. MannWhitney U test was used to compare two groups for continuous variables without normal distribution. The relationship between the preoperative independent variables and the development of PoAF was evaluated by a binary logistic regression analysis. For all tests, a $\mathrm{p}<0.05$ was considered statistically significant. Receiver-operating characteristic (ROC) curve was applied for the prediction of PoAF after off-pump CABG and the area under the curve was calculated for RDW and MPV levels.

\section{Results}

A total number of 24 patients in the $\mathrm{PoAF}(+)$ group $(66.7 \%$ male, mean age: $66 \pm 7.8$ years) and 69 patients in the PoAF(-) group ( $72.5 \%$ male, mean age: $56.26 \pm 11.53$ years) were enrolled in to the study. The demographic and clinical properties of the subjects are summarized in Table 1. Both $\operatorname{PoAF}(+)$ group and PoAF(-) group were similar to each other with regard to demographic characteristics. However, there was statistical difference between two group in terms of age $(p=0.000)$ (Tab. 1).

The comparisons of laboratory and operative parameters are shown in Table 2. Significant differences were observed between two groups in terms of hematocrit, RDW, blood urea nitrogen

Tab. 1. Demographic features of patients.

\begin{tabular}{lccc}
\hline & $\begin{array}{c}\text { PoAF }(-) \text { group } \\
\mathrm{n}=69\end{array}$ & $\begin{array}{c}\text { PoAF }(+) \text { group } \\
\mathrm{n}=24\end{array}$ & $\mathrm{p}^{*}$ \\
\hline Age (years) & $56.26 \pm 11.53$ & $66 \pm 7.8$ & $0.000^{*}$ \\
Male gender, n (\%) & $50(72.5)$ & $16(66.7)$ & $0.590^{*}$ \\
Hypertension, n (\%) & $32(46.4)$ & $15(62.5)$ & $0.174^{*}$ \\
Diabetes mellitus, n (\%) & $29(42)$ & $6(25)$ & $0.138^{*}$ \\
CAD, n (\%) & $10(14.5)$ & $6(25)$ & $0.240^{*}$ \\
PAD, n (\%) & $5(7.2)$ & $3(12.5)$ & $0.429^{*}$ \\
Preoperatif MI, n (\%) & $19(27.5)$ & $5(20.8)$ & $0.518^{*}$ \\
Beta-blocker therapy, n (\%) & $65(92.8)$ & $21(87.5)$ & $0.429^{*}$ \\
Statin therapy, n (\%) & $47(68.1)$ & $18(75)$ & $0.527^{*}$ \\
ACE-I/ARB therapy, n (\%) & $29(56.5)$ & $14(58.3)$ & $0.609^{*}$ \\
EuroSCORE II & $2.77 \pm 1.88$ & $3.67 \pm 1.9$ & $0.054^{\mathrm{a}}$ \\
Number of anastomosis & $1.35 \pm 0.56(1-3)$ & $1.63 \pm 0.71(1-3)$ & $0.067^{\mathrm{a}}$ \\
BSA & $1.87 \pm 0.17$ & $1.83 \pm 0.14$ & $0.265^{\#}$ \\
BMI & $27.31 \pm 4.51$ & $27.47 \pm 4.2$ & $0.735^{\mathrm{a}}$ \\
\hline PoAF - Pop & & &
\end{tabular}

PoAF - Postoperative atrial fibrillation, CAD - Carotid artery disease, PAD - Peripheral artery disease, ACE-I - Angiotensin-converting enzyme inhibitor, ARB Angiotensin-receptor blocker, BSA - Body surface area, BMI - Body mass index. * Pearson Chi- Suquare, " Student's $t$ test, ${ }^{a}$ Mann-Whitney U test. 
Tab. 2. Laboratory and operative variables.

\begin{tabular}{|c|c|c|c|}
\hline & $\begin{array}{c}\text { PoAF (-) group } \\
n=69\end{array}$ & $\begin{array}{c}\text { PoAF (+) group } \\
n=24\end{array}$ & $\mathrm{p}^{*}$ \\
\hline Hematocrit (\%) & $41.16 \pm 3.47$ & $38.83 \pm 3.36$ & $0.001^{a}$ \\
\hline White blood cell $\left(10^{3} / \mu \mathrm{L}\right)$ & $9.01 \pm 2.2$ & $9.12 \pm 2.39$ & $0.846^{\#}$ \\
\hline Platelet $\left(10^{3} / \mu \mathrm{L}\right)$ & $249.36 \pm 72.76$ & $248.17 \pm 86.7$ & $0.871^{\mathrm{a}}$ \\
\hline Red cell distribution width (\%) & $13.63 \pm 1.17$ & $14.52 \pm 1.3$ & $0.001^{\mathrm{a}}$ \\
\hline Mean platelet volume (fL) & $8.72 \pm 0.77$ & $9.03 \pm 0.84$ & $0.061^{\mathrm{a}}$ \\
\hline BUN (mg/dL) & $17.43 \pm 6.86$ & $22.12 \pm 7.88$ & $0.004^{\mathrm{a}}$ \\
\hline Creatinine (mg/dL) & $0.84 \pm 0.23$ & $1.01 \pm 0.24$ & $0.004^{\mathrm{a}}$ \\
\hline $\mathrm{Na}(\mathrm{mEq} / \mathrm{L})$ & $139.16 \pm 2.57$ & $138.88 \pm 2.36$ & $0.635^{\mathrm{a}}$ \\
\hline $\mathrm{K}(\mathrm{mEq} / \mathrm{L})$ & $4.05 \pm 0.68$ & $4.12 \pm 0.39$ & $0.724^{\mathrm{a}}$ \\
\hline $\mathrm{Ca}(\mathrm{mg} / \mathrm{dl})$ & $9.24 \pm 0.46$ & $9.01 \pm 0.52$ & $0.069^{\#}$ \\
\hline $\mathrm{Mg}(\mathrm{mg} / \mathrm{dl})$ & $1.9 \pm 0.22$ & $1.89 \pm 0.16$ & $0.813^{\#}$ \\
\hline Free $\mathrm{T}_{3}(\mathrm{pg} / \mathrm{mL})$ & $3.03 \pm 0.44$ & $2.86 \pm 0.3$ & $0.136^{\mathrm{a}}$ \\
\hline Free $\mathrm{T}_{4}(\mathrm{ng} / \mathrm{dL})$ & $1.15 \pm 0.21$ & $1.09 \pm 0.17$ & $0.169^{\#}$ \\
\hline $\mathrm{TSH}(\mathrm{IU} / \mathrm{mL})$ & $3.29 \pm 5.42$ & $2.33 \pm 1.33$ & $0.909^{\mathrm{a}}$ \\
\hline C Reactive protein (mg/dL) & $10.33 \pm 17.52$ & $12.57 \pm 21.77$ & $0.287^{\mathrm{a}}$ \\
\hline Total Cholesterol(mg/dl) & $196.49 \pm 34.86$ & $198.67 \pm 30.35$ & $0.787^{\#}$ \\
\hline LDL-C (mg/dl) & $121.94 \pm 31.57$ & $125.55 \pm 26.88$ & $0.618^{\#}$ \\
\hline HDL-C (mg/dl) & $40.7 \pm 5.96$ & $43.07 \pm 9.89$ & $0.306^{\mathrm{a}}$ \\
\hline $\mathrm{TG}(\mathrm{mg} / \mathrm{dl})$ & $171.96 \pm 75.2$ & $141.71 \pm 56.88$ & $0.075^{\mathrm{a}}$ \\
\hline Ejection fraction ( \%) & $48.91 \pm 11.37$ & $47.92 \pm 9.31$ & $0.538^{\mathrm{a}}$ \\
\hline Left atrium diameter $(\mathrm{mm})$ & $37.81 \pm 3.9$ & $38.46 \pm 4.58$ & $0.650^{\mathrm{a}}$ \\
\hline Postoperative bleeding (ml) & $597.1 \pm 233.08$ & $620 \pm 213.03$ & $0.644^{\mathrm{a}}$ \\
\hline ICU stay (day) & $2.14 \pm 0.43$ & $2.79 \pm 0.77$ & $0.000^{\mathrm{a}}$ \\
\hline Hospital stay (day) & $6.01 \pm 0.93$ & $7.25 \pm 1.26$ & $0.000^{\mathrm{a}}$ \\
\hline
\end{tabular}

PoAF - Postoperative atrial fibrillation, ICU - Intensive care unit. "Student's-t test, ${ }^{a}$ Mann-Whitney U test.

(BUN), creatinin, ICU stay and hospital stay. There were statistical differences between the two groups in terms of hematocrit, RDW, BUN, creatinin, ICU stay and hospital stay as a negative affect to $\operatorname{PoAF}(+)$ group $(\mathrm{p}=0.001, \mathrm{p}=0.001, \mathrm{p}=0.004, \mathrm{p}=0.004, \mathrm{p}=$ 0.000 and $p=0.000$, respectively) (Tab. 2). In addition, ejection fraction, left atrium diameter and MPV level were not significantly different between the groups $(p=0.538, p=0.650$ and $p=0.061$ respectively) (Tab. 2).

Risk factors related to the development of PoAF were included in the univariate logistic regression analysis. In unadjusted univariate logistic regression analysis, the PoAF was significantly correlated with age (OR [Odds Ratio]: 1.083, $95 \%$ CI [Confidence

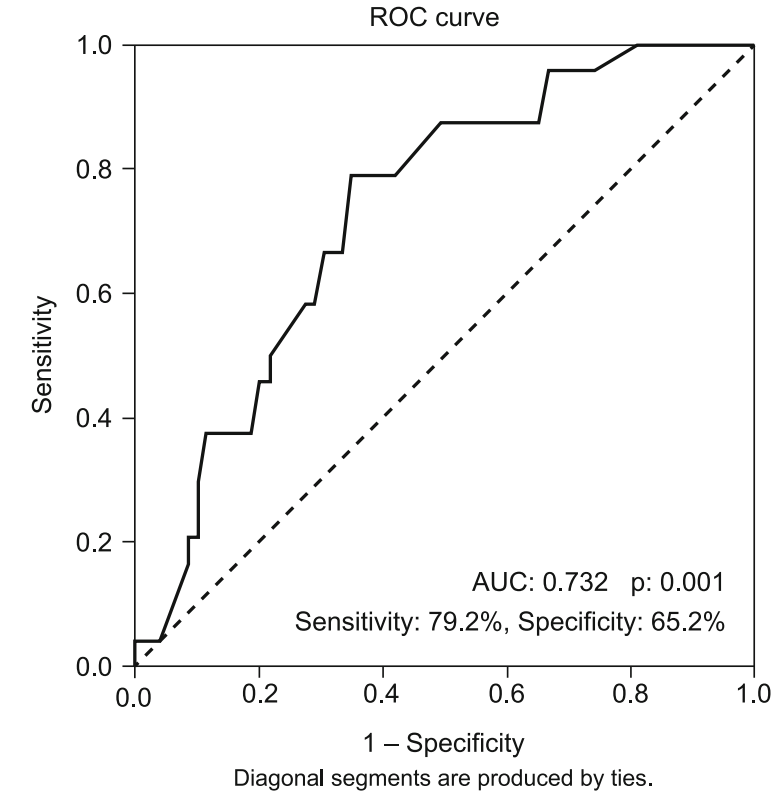

Fig. 1. ROC curve and AUC for RDW for predicting PoAF. ROC Receiver operation characteristic, AUC - Area under the curve, RDW - Red cell distribution witdh, PoAF - Postoperative atrial fibrillation.

interval]: $1.025-1.144, \mathrm{p}=0.004)$, hematocrit (OR: 0.829, $95 \%$ CI: $0.720-0.956, p=0.010)$, RDW (OR: $1.740,95 \%$ CI: $1.166-$ $2.598, \mathrm{p}=0.007)$, BUN (OR: 1.084, $95 \%$ CI: 1.018-1.154, p = 0.011 ) and creatinine (OR: 16.313, $95 \%$ CI: 2.193-121.333, $\mathrm{p}=$ 0.006 ) but was not correlated with hypertension, diabetes mellitus, ejection fraction, left atrium diameter, EuroSCORE II and MPV (Tab. 3). Only age was identified as an independent predictor of development AF after off-pump CABG surgery in multivariate analysis (OR: 1.074, 95 \% CI: 1.016-1.135, p=0.012) (Tab. 3).

Additionally, in ROC curve analysis, it was determined a cutoff level of 13.65 for RDW for predicting PoAF (Area under the curve (AUC): $0.732,95 \%$ CI: $0.625-0.840, \log$ rank $\mathrm{p}=0.001$ )

Tab. 3. Binary Logistic regression analysis to identify predictors of PoAF.

\begin{tabular}{|c|c|c|c|c|c|c|}
\hline \multirow[b]{2}{*}{ Variables } & \multicolumn{3}{|c|}{ Univariate analysis } & \multicolumn{3}{|c|}{ Multivariate analysis } \\
\hline & $\mathrm{p}$ & $\begin{array}{c}\operatorname{Exp}(\mathrm{B}) \\
\text { Odds Ratio }\end{array}$ & $\begin{array}{c}95 \% \text { C.I. } \\
\text { Lower Upper }\end{array}$ & $\mathrm{p}$ & $\begin{array}{c}\operatorname{Exp}(\mathrm{B}) \\
\text { Odds Ratio }\end{array}$ & $\begin{array}{c}95 \% \text { C.I. } \\
\text { Lower Upper }\end{array}$ \\
\hline Age & 0.001 & 1.089 & $1.306-1.145$ & 0.012 & 1.074 & $1.016-1.135$ \\
\hline HT & 0.177 & .519 & $.200-1.345$ & & & \\
\hline DM & 0.143 & 2.175 & $.769-6.155$ & & & \\
\hline $\mathrm{EF}$ & 0.697 & .992 & $.950-1.035$ & & & \\
\hline LAD & 0.502 & 1.041 & $.926-1.171$ & & & \\
\hline EuroSCORE II & 0.054 & 1.274 & $.996-1.629$ & & & \\
\hline Hct & 0.010 & .829 & $.720-.956$ & 0.125 & .866 & $.721-1.041$ \\
\hline RDW & 0.007 & 1.740 & $1.166-2.598$ & 0.094 & 1.519 & $.931-2.479$ \\
\hline MPV & 0.103 & 1.603 & $.909-2.824$ & & & \\
\hline BUN & 0.011 & 1.084 & $1.018-1.154$ & 0.214 & 1.062 & $.966-1.168$ \\
\hline Creatinine & 0.006 & 16.313 & $2.193-121.333$ & 0.475 & 3.009 & $.147-61.792$ \\
\hline
\end{tabular}

PoAF - Postoperative atrial fibrillation, , HT; Hypertension, DM; Diabetes mellitus, EF; Ejection fraction, LAD; Left atrium diameter, RDW - Red cell distribution, MPV - Mean platelet volume, Hct - Hematocrit 


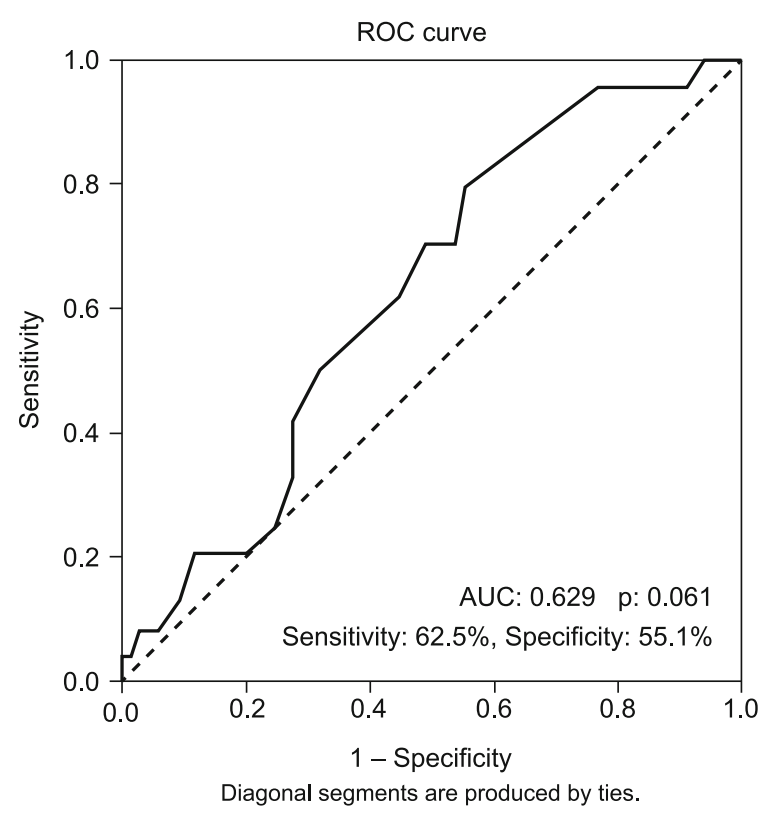

Fig. 2. ROC curve and AUC for MPV for predicting PoAF. ROC Receiver operation characteristic, AUC - Area under the curve, MPV - Mean platelet volume, PoAF - Postoperative atrial fibrillation.

(Fig. 1). For MPV, it was determined as a cut-off level of 8.75 for predicting PoAF (AUC: 0.629, 95 \% CI: 0.508-0.749, Log rank $p=0.062$ ) (Fig. 2). For predicting PoAF, in the measurements above their cut-off values, there was $79.2 \%$ sensitivity and $65.2 \%$ specificity for RDW and $62.5 \%$ sensitivity and $55.1 \%$ specificity for MPV.

\section{Discussion}

In our study, we assessed the effect of RDW and MPV levels in the development of PoAF in patients undergoing off-pump CABG. In univariate logistic regression analysis, we found that advanced age, lower hematocrit levels, higher RDW, higher BUN and creatinine levels were significantly correlated with the development of PoAF. In multivariate logistic regression analysis, only advanced age was detected as an independent predictor for the development of POAF. In addition, in the measurements above cut-off value, there was $79.2 \%$ sensitivity and $65.2 \%$ specificity for predicting PoAF for RDW (AUC: 0.732, Log rank p: 0.001), $62.5 \%$ sensitivity and $55.1 \%$ specificity for MPV (AUC: 0.629, Log rank p: 0.062). But in our study, RDW and MPV were not independent predictors for the development of PoAF.

The incidence of PoAF following CABG surgery is seen in $25-40 \%$ of cases (2). However, its frequency reaches $62 \%$ following combined CABG and valve surgery (11). Previous study demonstrated that patients with mitral regurgitation were more likely to experience recurrent AF post-ablation (12). Therefore, we excluded 7 patients with moderate mitral valve disease so that it is not effecting the outcome of the study.
There has been an increased risk of new-onset $\mathrm{AF}$ in patients with COPD. Mathew et al (13) have showed that COPD increased the incidence of both persistent and paroxysmal AF and the incidence of PoAF increased to $43 \%$ in the presence of COPD. Negative prognostic effect of atrial fibrillation has been demonstrated in COPD patients. For this reason, patients with COPD were excluded in order to have more accurate results.

Chronic renal disease is associated with the risk of multiple life threatening complications and AF is common in this group of patients. In the large population-based study, Alonso et al (14) reported that reduced renal function and presence of albuminuria were strongly associated with the incidence of AF independently of other risk factors. Similar to these studies, in our study, higher BUN and higher creatinine levels were statistically significant in patients with PoAF. In addition, BUN and creatinine levels were significantly correlated with the development of PoAF in univariate logistic regression analysis. Our study showed once again a relationship between reduced renal function and $\mathrm{AF}$.

In our study, both in univariate logistic regression analysis and in multivariate logistic regression analysis, we found that only age significantly correlated with the development of PoAF. Advanced age was detected as an independent predictor of the development of AF in our study. Age-related changes, including atrial fibrosis and accumulation of amyloid, can cause intraatrial reentry, which leads to the development of AF (15). Age has been repeatedly shown to be the major risk factor for AF after cardiac surgery $(16,17)$. Our results were generally consistent with the literature. When the age is considered as a risk factor, it is known that elder patients have high risk for developing AF.

In the last decade, studies have been carried out on prognostic marker of RDW in various cardiovascular disorders besides blood diseases. A variety of mechanisms have been proposed for the association between RDW levels and cardiovascular outcomes. Oxidative stress and chronic inflammation may reduce survival of erythrocytes leading to a more mixed population of erythrocyte volumes in circulation $(18,19)$. In acute and chronic cardiovascular diseases, inflammatory cytokines and neurohumoral mediators are activated. Inflammatory cytokines have been found to suppress the maturation of erythrocytes, so the increased immature erythrocytes may reflect higher RDW values (19). It has been reported that an exaggerated inflammatory response is associated with a higher risk of PoAF (20). Previous studies have demonstrated that RDW is independently associated with both AF and postoperative newonset AF following elective cardiac surgery with a high sensitivity and specificity $(21,22)$. In a systematic review with meta-analysis, mean RDW was $15.03 \%$ in the $\operatorname{PoAF}(+)$ group and $14.62 \%$ in the $\mathrm{PoAF}(-)$ group and the meta analysis showed that RDW was significantly higher in the $\operatorname{PoAF}(+)$ group as a positive predictor $(\mathrm{p}<0.001)(23)$. In a previous study, Eryd et al $(21)$ demonstrated that high RDW values were associated with the development of paroxysmal AF. Gungor et al (24) reported that RDW is an independent marker of non-valvular $\mathrm{AF}$ in multivariate logistic regression analysis (OR: 4.18, $95 \%$ CI 2.15-8.15, $\mathrm{p}=0.01$ ). In one of the first studies to investigate the relationship between RDW and PoAF in patients undergoing CABG, Ertas et al (8) found that 
while preoperative RDW levels were significantly higher in patients who developed AF, there was not any correlation between postoperative RDW levels and AF. In addition, they remarked that preoperative RDW level predicts new-onset AF after CABG in patients without histories of $\mathrm{AF}$ (cutpoint of $13.45,61 \%$ sensitivity, $60 \%$ specificity) (8). Likewise, we think that postoperative RDW value can not be associated with atrial fibrillation after CABG due to possible bleeding, hypoxia, and inotropic agent use. Therefore, we assessed only preoperative RDW values. In our study, we found that RDW levels were significantly higher in the PoAF $(+)$ group $(p=0.001)$. Also, in our study, in univariate logistic regression analysis we found that higher RDW levels were correlated with the development of PoAF (OR: 1.740, $95 \%$ CI: 1.166-2.598, p $=0.007)$. But, RDW levels were not an independent predictor for the development of PoAF. For predicting PoAF above cut-off level of 13.65 of RDW , in ROC curve analysis, we found that there was $79.2 \%$ sensitivity and $65.2 \%$ specificity (AUC: $0.732,95 \%$ CI: $0.625-0.840, \log$ rank $p=0.001$ ) (Fig. 1). There is no study indicating the relationship between the RDW levels and PoAF in patients undergoing isolated off-pump CABG. Therefore, the results of our study may take into consideration relationship between high RDW levels and postoperative AF.

Activated platelets have larger volumes and contain wide range of vasoactive substances and prothrombotic factors. Therefore, MPV may also be a response to inflammation and thrombosis (9). It has been reported that an exaggerated inflammatory response is associated with a higher risk of PoAF (20). An increased MPV is associated with overall cerebrovascular and cardiovascular mortality rates (10). Previous studies have shown the association of MPV with thrombotic events (9), slow coronary flow (25) and left atrial stasis (10) in patients with AF. In a systematic review with meta-analysis, mean of MPV was 9.35 femtolitre(fl) in the PoAF(+) group and $9.05 \mathrm{fl}$ in the PoAF(-) group and the meta analysis showed that MPV was significantly higher in the PoAF $(+)$ group as a positive predictor $(\mathrm{p}<0.001)(23)$. In a study evaluating the relationship between serum laboratory parameters and new onset atrial fibrillation, Karatas et al.(26) found that increased levels of CRP, MPV, RDW, uric acid and neutrophil to lymphocyte ratio independently predicted new onset AF. In previous study to investigate the relationship between MPV and PoAF in patients undergoing $\mathrm{CABG}$, Erdem et al (27) found that MPV was significantly higher in the AF group $(\mathrm{p}<0.001)$ and in multivariate logistic regression analysis showed that MPV were independent predictors of PoAF (95 \% CI: 1.326-4.958, p: 0.005). In addition, they remarked their results show that increased platelet activity is associated with the development of AF after CABG. In our study, we found that MPV was not significantly higher in the $\mathrm{PoAF}(+)$ group $(\mathrm{p}=0.061)$. In addition, in univariate logistic regression analysis we found that higher MPV was not correlated with the development of PoAF $(\mathrm{p}=0.103)$. Therefore, MPV was not an independent predictor for the development of PoAF. But, in ROC curve analysis, we found that there was $62.5 \%$ sensitivity and $55.1 \%$ specificity for MPV (AUC: 0.629, Log rank p =0.062) (Fig. 2). We think that our AUC value is not a bad value for MPV. But there is a need for further work in this issue.
In our study, it provided homogeneity between the groups, because we excluded the risk factors such as COPD and valvular heart diseases for developing AF. Therefore, the results of our study may be more specific in terms of the relationship between raised RDW level and raised MPV with PoAF.

\section{Conclusion}

There is no study on the relationship between the MPV and PoAF in patients undergoing isolated off-pump CABG. Many factors contribute to the development of AF after coronary bypass surgery. Many studies have been done on PoAF development. RDW levels may be a predictor of postoperative complications such as AF. As a result of this study, we thought that high RDW level could be a factor of the development of PoAF and there is a need for further work for MPV. We think that these factors should be taken into account before surgery.

\section{References}

1. Guenancia C, Pujos C, Debomy F, Malapert G, Laurent G, Bouchot O. Incidence and Predictors of New-Onset Silent Atrial Fibrillation after Coronary Artery Bypass Graft Surgery. Biomed Res Int 2015; 2015 : 703685. doi: 10.1155/j.bri.2015/703685, indexed in Pubmed: 26290873.

2. Júnior MSB, Matkovski PD, Di Giovanni FJ, Fenili R, Varella EL, Dietrich A. Incidence of postoperative atrial fibrillation in patients undergoing on-pump and off-pump coronary artery bypass grafting. Braz J Cardiovasc Surg 2015; 30: 316-324.

3. Fo“rhe'cz Z, Gombos T, Borgulya G, Pozsonyi Z, Proha'szka Z, Ja'noskuti L. Prediction of clinical events and relationship with markers of ineffective erythropoiesis, inflammation, renal function, and nutritional state. Am Heart J 2009; 158: 659-666.

4. Allen LA, Felker GM, Mehra MR et al. Validation and potential mechanisms of red cell distribution width as a prognostic marker in heart failure. J Card Fail 2010; 16: 230-238.

5. Ani C, Ovbiagele B. Elevated red blood cell distribution width predicts mortality in persons with known stroke. J Neurol Sci 2009; 277: 103-108.

6. Dabbah S, Hammerman H, Markiewicz W, Aronson D. Relation between red cell distribution width and clinical outcomes after acute myocardial infarction. Am J Cardiol 2010; 105: 312-317.

7. Horne BD, May HT, Kfoury AG et al. The Intermountain Risk Score (including the red cell distribution width) predicts heart failure and other morbidity endpoints. Eur J Heart Fail 2010; 12: 1203-1213.

8. Ertas G, Aydin C, Sönmez O et al. Red cell distribution width predicts new-onset atrial fibrillation after coronary artery bypass grafting. Scand Cardiovasc J 2013; 47: 132-135.

9. Xu XF, Jiang FL, Ou MJ, Zhang ZH. The association between mean platelet volume and chronic atrial fibrillation and the presence of thrombotic events. Biomed Rep. 2015; 3: 388-394.

10. Providência R, Faustino A, Paiva L et al. Mean platelet volume is associated with the presence of left atrial stasis in patients with non-valvular atrial fibrillation. BMC Cardiovasc Disord. 2013 10; 13: 40

11. Maisel WH, Rawn JD, Stevenson WG. Atrial fibrillation after cardiac surgery. Ann Intern Med 2001; 135: 1061-1073. 


\section{5-340}

12. Zhao L, Jiang W, Zhou $\mathbf{L}$ et al. The role of valvular regurgitation in catheter ablation outcomes of patients with long-standing persistent atrial fibrillation. Europace 2014; 16: 848-854.

13. Mathew JP, Fontes ML, Tudor IC et al. Investigators of the Ischemia Research and Education Foundation; Multicenter Study of Perioperative Ischemia Research Group. A multicenter risk index for atrial fibrillation after cardiac surgery. JAMA 2004; 291: 1720-1729.

14. Franczyk B, Gluba-Brzózka A, Ciałkowska-Rysz A, Banach M, Rysz J. The Problem of Atrial Fibrillation in Patients with Chronic Kidney Disease. Curr Vasc Pharmacol. 2016; 14: 260-265.

15. Nisanoglu V, Erdil N, Aldemir M et al. Atrial fibrillation after coronary artery bypass grafting in elderly patients: incidence and risk factor analysis. Thorac Cardiovasc Surg 2007; 55: 32-38.

16. Leitch JW, Thomson D, Baird DK, Harris PJ. The importance of age as a predictor of atrial fibrillation and flutter after coronary artery bypass grafting. J Thorac Cardiovasc Surg 1990; 100: 338-342.

17. Turk T, Vural H, Eris C, Ata Y, Yavuz S. Atrial fibrillation after offpump coronary artery surgery: a prospective, matched study. J Int Med Res 2007; 35: 134-142.

18. Kiefer CR, Snyder LM. Oxidation and erythrocyte senescence. Curr Opin Hematol 2000; 7: 113-116.

19. Weiss G, Goodnough LT. Anemia of chronic disease. N Engl J Med 2005; 352: 1011-1023.

20. Aviles RJ, Martin DO, Apperson-Hansen C et al. Inflammation as a risk factor for atrial fibrillation. Circulation 2003; 108: 300-310 .
21. Adamsson ES, Borne Y, Melander O et al. Red blood cell distribution width is associated with incidence of atrial fibrillation. J Intern Med 2014; 275: 84-92.

22. Gerede DM, Kaya CT, Vurgun VK et al. Red cell distribution width as a predictor of left atrial spontaneous echo contrast in echocardiography. Medicine 2015; 94 : e712.

23. Weymann A, Ali-Hasan-Al-Saegh S, Popov AF et al. Hematologic indices as predictors of atrial fibrillation following isolated coronary artery bypass grafting, valvular surgery or combined procedures: A systematic review with meta-analysis. Kardiol Pol 2018; 76 (1): 107-118.

24. Güngör B, Özcan KS, Erdinler I et al. Elevated levels of RDW is associated with non-valvular atrial fibrillation. J Thromb Thrombolysis 2014; 37: 404-410.

25. Feng C, Mei W, Luo $\mathbf{C}$ et al. Relationship between mean platelet volume and coronary blood flow in patients with atrial fibrillation. Heart Lung Circ 2013; 22: 43C49.

26. Karatas MB, Canga $Y$, Ipek $\mathbf{G}$ et al. Association of admission serum laboratory parameters with new-onset atrial fibrillation after a primary percutaneous coronary intervention. Coron Artery Dis 2016; 27: $128-134$.

27. Erdem K, Ayhan S, Ozturk S et al. Usefulness of the mean platelet volume for predicting new-onset atrial fibrillation after isolated coronary artery bypass grafting. Platelets 2014; 25: 23-26.

Received February 14, 2018. Accepted March 5, 2018. 